\title{
Transperineal-incision urethrectomy combined with laparoscopic prostatectomy for a male patient with squamous cell carcinoma involving distal plus proximal urethra and untypical symptoms -a case report
}

\author{
Miao Wang ${ }^{1,2 \#}$, Mingzi Yang ${ }^{3 \#}$, Pengjie Wu ${ }^{1}$, Shumin Deng ${ }^{1}$, Jianlong Wang ${ }^{1}$, Jia Chen ${ }^{1}$, Jianye Wang ${ }^{1}$, \\ Ming Liu ${ }^{1}$ \\ ${ }^{1}$ Department of Urology, Beijing Hospital, National Center of Gerontology, Institute of Geriatric Medicine, Chinese Academy of Medical Sciences, \\ Beijing, China; ${ }^{2}$ Graduate School of Peking Union Medical College, Chinese Academy of Medical Sciences, Beijing, China; ${ }^{3}$ Plastic Surgery \\ Hospital, Chinese Academy of Medical Sciences and Peking Union Medical College, Beijing, China \\ \#These authors contributed equally to this work. \\ Correspondence to: Ming Liu. No. 1 Dongdan Dahua Road, Dongcheng District, Beijing 100730, China. Email: liumingbjh@126.com.
}

\begin{abstract}
Primary urethral carcinoma (PUC) is a rare malignancy, covering less than $1 \%$ of all genitourinary cancers. Different tumor location, classified as tumor in distal or proximal urethra, represents different characteristics and often leads to different treatment modality. However, data on the surgical approach for PUC involving both distal and proximal urethra remains rare. In this case, we presented a 75 -year-old man with untypical symptoms of perineal mass and unspecific frequent and painful urination. Results of multiparametric magnetic resonance imaging (mp-MRI), positron emission tomography/computed tomography (PET/CT) scan, and percutaneous biopsy revealed a cT2N1M0 PUC involving both distal and proximal urethra. Given the request of patients for a normal penile appearance after surgery, a transperinealincision urethrectomy combined with laparoscopic prostatectomy and iliac lymphadenectomy was performed with optimal outcomes. The results of histopathological analysis revealed a moderately-high differentiated PUC with no positive lymph node. Post-operative recovery was uneventful. On first visit 1-month after surgery, physical examination revealed a satisfactory wound healing and appearance of penis and no recurrent lesions were found on mp-MRI. This is a rare case with untypical symptoms indicating that patients with PUC involving both distal and proximal urethra may present with no symptoms of urethral stricture but only non-specific lower urinary symptoms. The surgical approach we proposed in this case proves to be a safe and feasible one to completely resect the tumor and preserve a normal appearance of penis, thus worth to be applied in the specific patient population.
\end{abstract}

Keywords: Urethral neoplasms; urethra; squamous cell carcinoma; case report

Submitted Jun 09, 2020. Accepted for publication Nov 12, 2020.

doi: $10.21037 /$ tau-20-984

View this article at: http://dx.doi.org/10.21037/tau-20-984

\section{Introduction}

Primary urethral carcinoma (PUC) is a rare malignancy, accounting for less than $1 \%$ of all genitourinary cancers (1). Only a small proportion of PUC originated in distal urethra with the majority in proximal urethra (2). PUC originated in different location usually has different histology, oncologic characteristics and prognosis, thus often calls for different treatment modality (3-5). The European Association of Urology (EAU) guidelines on PUC made clear recommendations for PUC in distal urethra and urothelial carcinoma (UC) of the prostate, however, no treatment options were offered in the current guidelines on PUC 


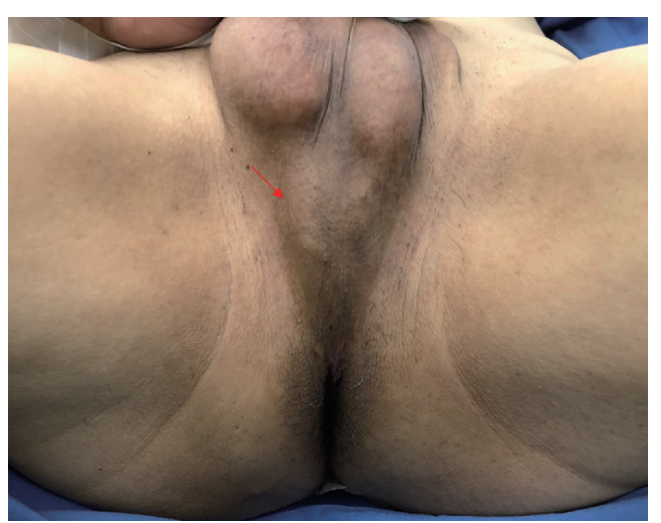

Figure 1 The appearance of tumor on physical examination before surgery. An approximately $3 \mathrm{~cm} \times 6 \mathrm{~cm}$ mass was palpable at perineum, firm and unmovable, with normal skin appearance (red arrow).

involving both distal and proximal urethra (6), leaving no standardized treatment modality. In this case, we presented a male patient with untypical symptoms diagnosed of PUC involving both distal and proximal urethra and a feasible surgical approach for treatment. We present the following article in accordance with the CARE Guidelines (7) and the CARE reporting checklist (available at http://dx.doi. org/10.21037/tau-20-984).

\section{Case presentation}

A 75 -year-old man presented with a palpable mass in the perineal region, with frequent and painful urination and a transient swelling and pain 2 months ago. These symptoms relieved several days after onset without any medication. Then the patient noticed a growth of the mass from about 2 to $6 \mathrm{~cm}$ in diameter in two months. No visible hematuria, bloody urethral discharge, dysuria, pelvic pain or fever presented during the course of the disease. Medical history of the patient included diabetes mellitus and hypothyroidism. On physical examination, an approximately $3 \mathrm{~cm} \times 6 \mathrm{~cm}$ mass was palpable at perineum, firm and unmovable, with normal skin appearance (shown as Figure 1). Digital rectal examination found no nodule within the prostate gland. No palpable superficial lymph node was found.

Urinalysis demonstrated red and white blood cells, while the result of urine culture was negative. Urine cytology identified squamous cell carcinoma (SCC). Results of other laboratory tests revealed elevated SCC antigen of $6.0 \mathrm{ng} / \mathrm{mL}$ (reference range $0-1.5 \mathrm{ng} / \mathrm{mL}$ ) and carcinoembryonic antigen (CEA) of $6.2 \mathrm{ng} / \mathrm{mL}$ (reference range $0-5 \mathrm{ng} / \mathrm{mL}$ ). A multiparametric magnetic resonance imaging (mpMRI) of pelvis showed a mass adjacent to the ventral side of proximal corpus spongiosum with irregular abnormal signal. Approximately $6.8 \mathrm{~cm} \times 3.7 \mathrm{~cm} \times 3.1 \mathrm{~cm}$ in diameter, the mass showed equal signal on T1WI and high signal on T2WI, with an unclear boundary with proximal urethra. A positron emission tomography/computed tomography (PET/CT) scan showed an increased radioactivity uptake of the mass beneath proximal corpus spongiosum with a SUVmax of 21.7 , and para-iliac lymph node (LN) enlargement on the right side $(1.8 \mathrm{~cm} \times 1.1 \mathrm{~cm})$, with a SUVmax of 6.1. Part of images of PET/CT and mp-MRI are shown in Figure 2. On cystoscopy, erosive mucosa was found covering a range from distal urethra (approximately 8 $\mathrm{cm}$ from urethral meatus) up to the apex of prostate gland. No obvious stricture of urethra was found via cystoscopy. Percutaneous biopsy of the perineal mass demonstrated a squamous cell carcinoma (SCC), moderately-high differentiation.

A radical operation was performed, including transperineal-incision urethrectomy, laparoscopic prostatectomy with pelvic lymphadenectomy (LND), and suprapubic cystostomy (Figure 3). A F18 Foley catheter was inserted before surgery. A midline incision was made in the perineal region beneath the penoscrotal junction of scrotum. The corpus spongiosum was carefully separated, proximally to the crus penis and distally to the glans penis. An integral resection of corpus spongiosum consisting of the complete tumor was performed with the corpus cavernous and dorsal neurovascular bundle uninjured and glans preserved. A laparoscopic prostatectomy combined with pelvic LND was performed at the same time. The extent of excision included LNs within external iliac region, internal iliac region, and obturator fossa. The bladder neck was closed after prostatectomy and a suprapubic cystostomy was performed. Indwelling tubes were placed for pelvic and perineal drainage which were removed four and ten days after surgery, respectively. The histopathologic results confirmed moderately-high differentiated SCC of urethra, with the apex of prostate infiltrated. All margins were negative. No metastasis was found in bilateral pelvic LNs (Figure 4). Human papillomavirus (HPV) was negative of the tumor.

Post-operative recovery was uneventful with only mild edema of perineal region and pelvic lymphorrhagia recovered without intervention. On first visit 1-month 


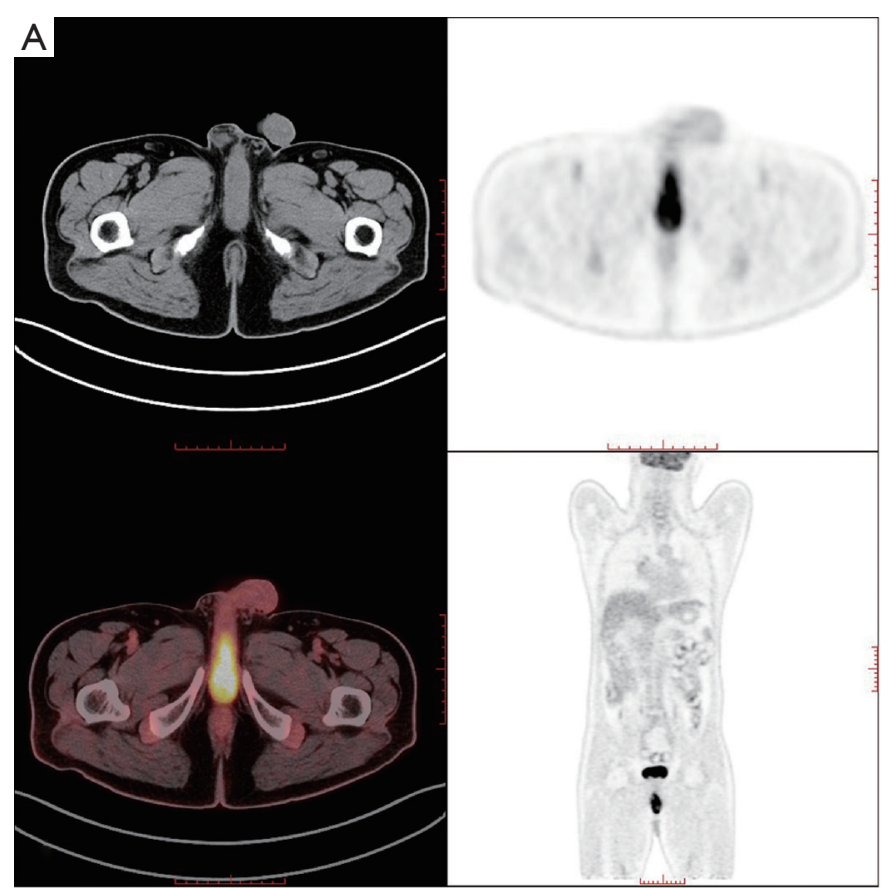

C

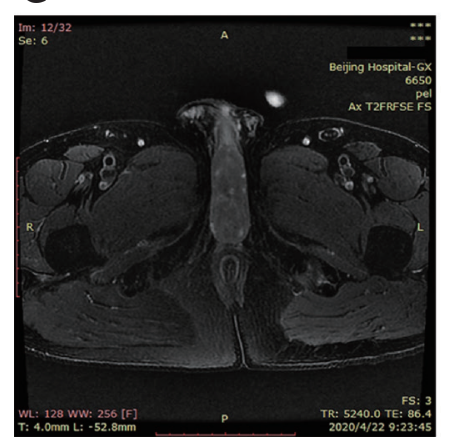

D

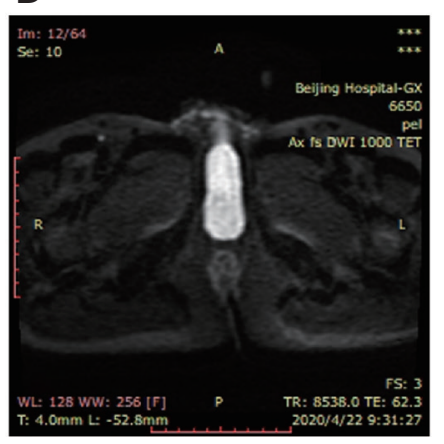

B

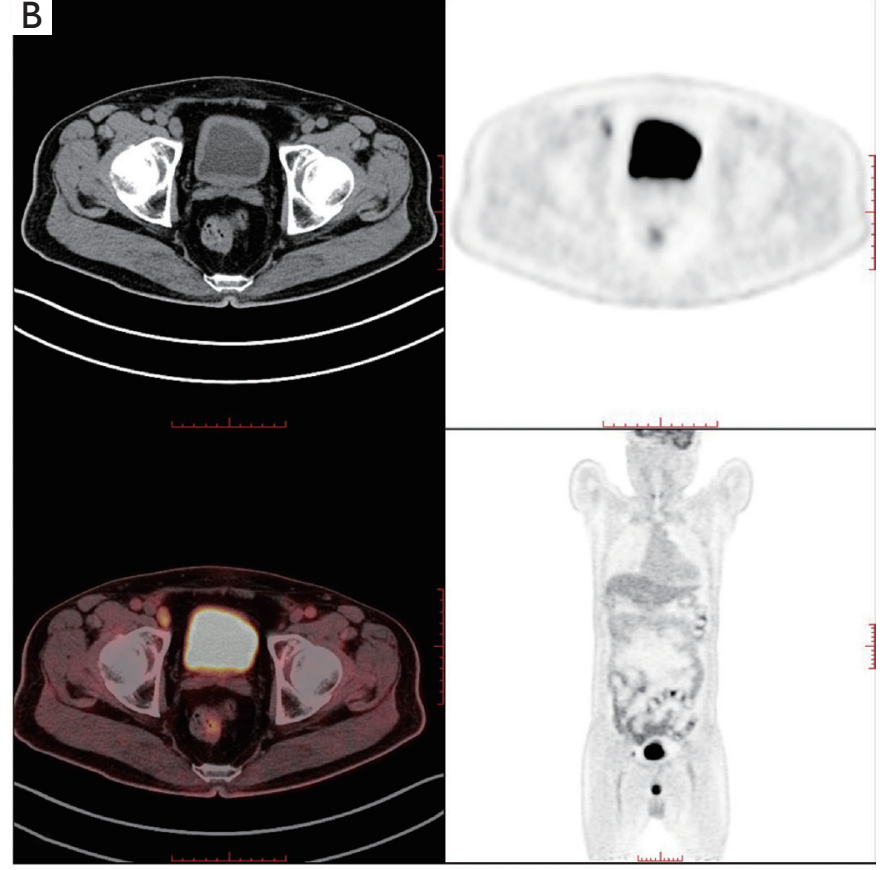

E

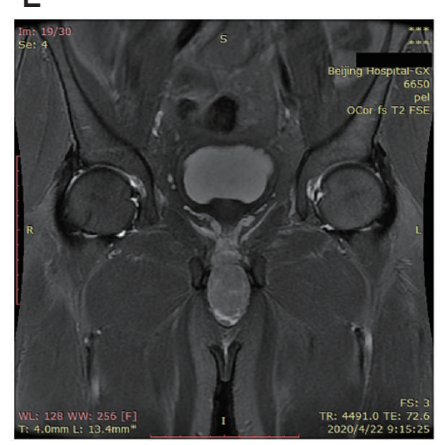

$\mathrm{F}$

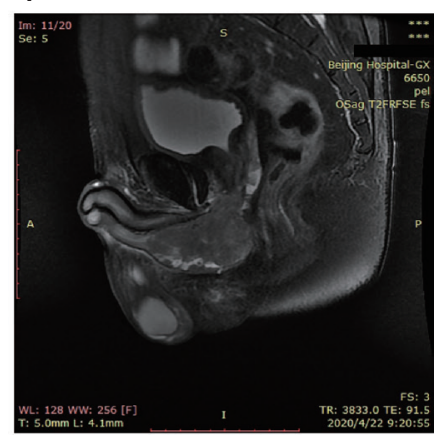

Figure 2 Pre-operation imaging data. Positron emission tomography/computed tomography (PET/CT) revealed (A) an increased radioactivity uptake of the mass beneath proximal corpus spongiosum with a SUVmax of 21.7 and (B) a para-iliac LN enlargement; multiparametric magnetic resonance imaging (mp-MRI) revealed a mass of no clear boundary with proximal urethra with a high signal on (C) axial T2WI, (D) axial DWI, (E) coronal T2WI, (F) sagittal T2WI.

after surgery, physical examination revealed a satisfactory wound healing and appearance of penis. No recurrent lesions were found on mp-MRI. The permanent bladder stoma functioned well. The patient was satisfactory with operation results and gradually got used to the permanent bladder stoma. The timeline of the medical history and the management the patient received was shown in Figure 5.

All procedures performed in studies involving human participants were in accordance with the ethical standards of the Institutional Review Board of Beijing Hospital and with the Helsinki Declaration (as revised in 2013). Written informed consent was obtained from the patient.

\section{Discussion}

PUC is a rare malignancy, covering less than $1 \%$ of all genitourinary cancers (1). The reported annual incidence of male and female PUC is 4.3 and 1.5 per-million population in a study based on data from the Surveillance, Epidemiology, and End Results (SEER) database (2). 

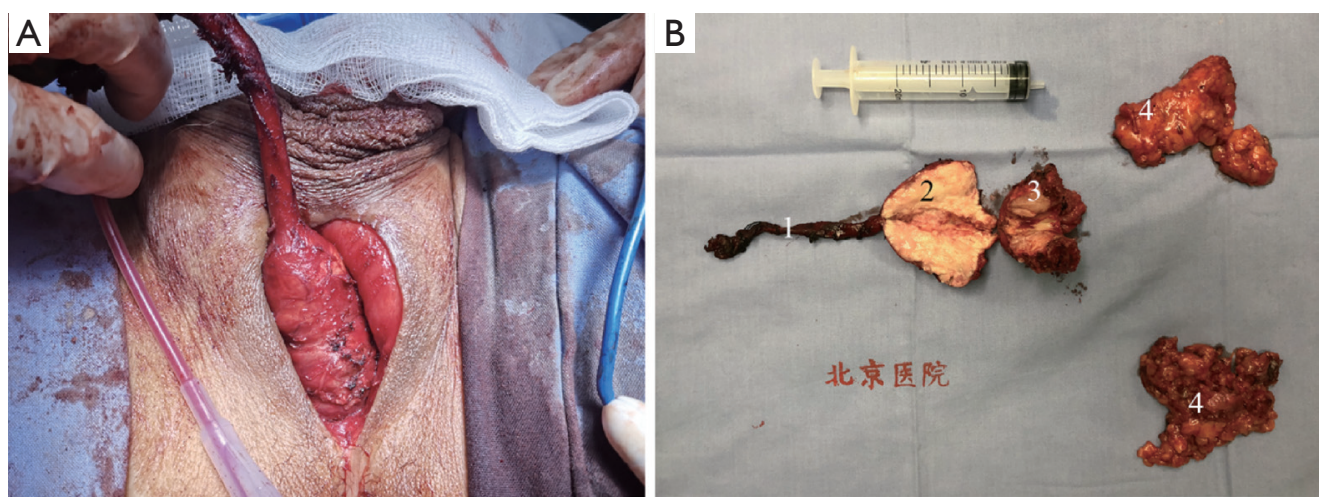

Figure 3 The transperineal incision and removed specimens. (A) A midline incision was made in the perineal region beneath the penoscrotal junction of scrotum by which the corpus spongiosum was integrally separated. (B) Prostate [3], lymph nodes [4] and integral corpus spongiosum [1] consisting complete tumor [2] resected.

A

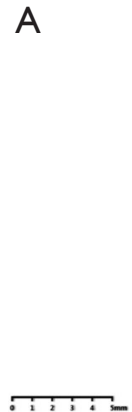

C

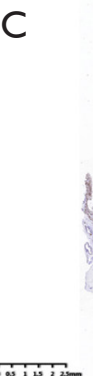

क्षisis

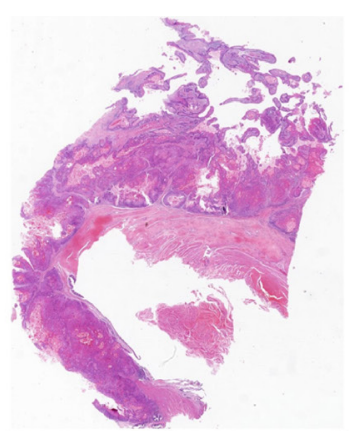

B

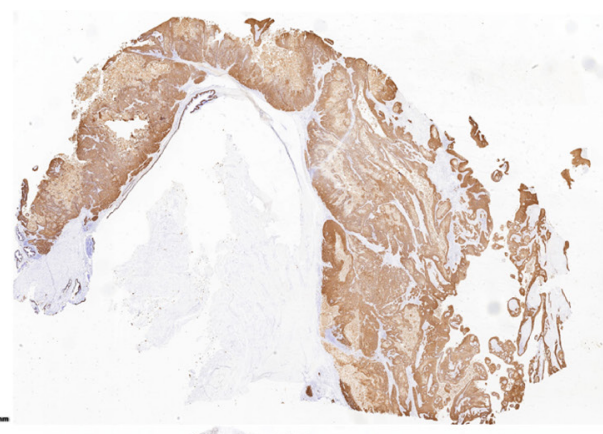

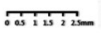

D

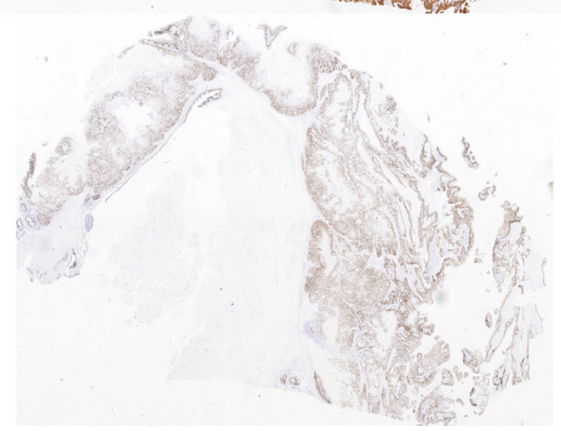

Figure 4 Pathologic and immunohistochemical (IHC) findings. Representative microscopic images of primary urethral squamous cell carcinoma (SCC) using hematoxylin and eosin staining was shown in (A). The IHC showed (B) CK5+, (C) P63+, and (D) Ki-67+.

Traditionally, SCC was thought to be the predominant histological type. However, some population-based studies published recently suggested UC (30-78\%) as the most common histology, followed by SCC (12-29\%) and adenocarcinoma (AC, 5-29\%) (2,6,8-11). When stratified by tumor location, SCC was the predominant histology in distal urethral while UC is the most common type in proximal urethral (2).
Various risk factors including urethral strictures, chronic irritation after intermittent catheterization/ urethroplasty, external beam radiation therapy, radioactive seed implantation, and chronic inflammation/urethritis following sexually transmitted diseases like HPV were reported according to EAU guidelines on PUC (6). The most common symptoms reported were gross hematuria, bloody urethral discharge for initial presentation, while 


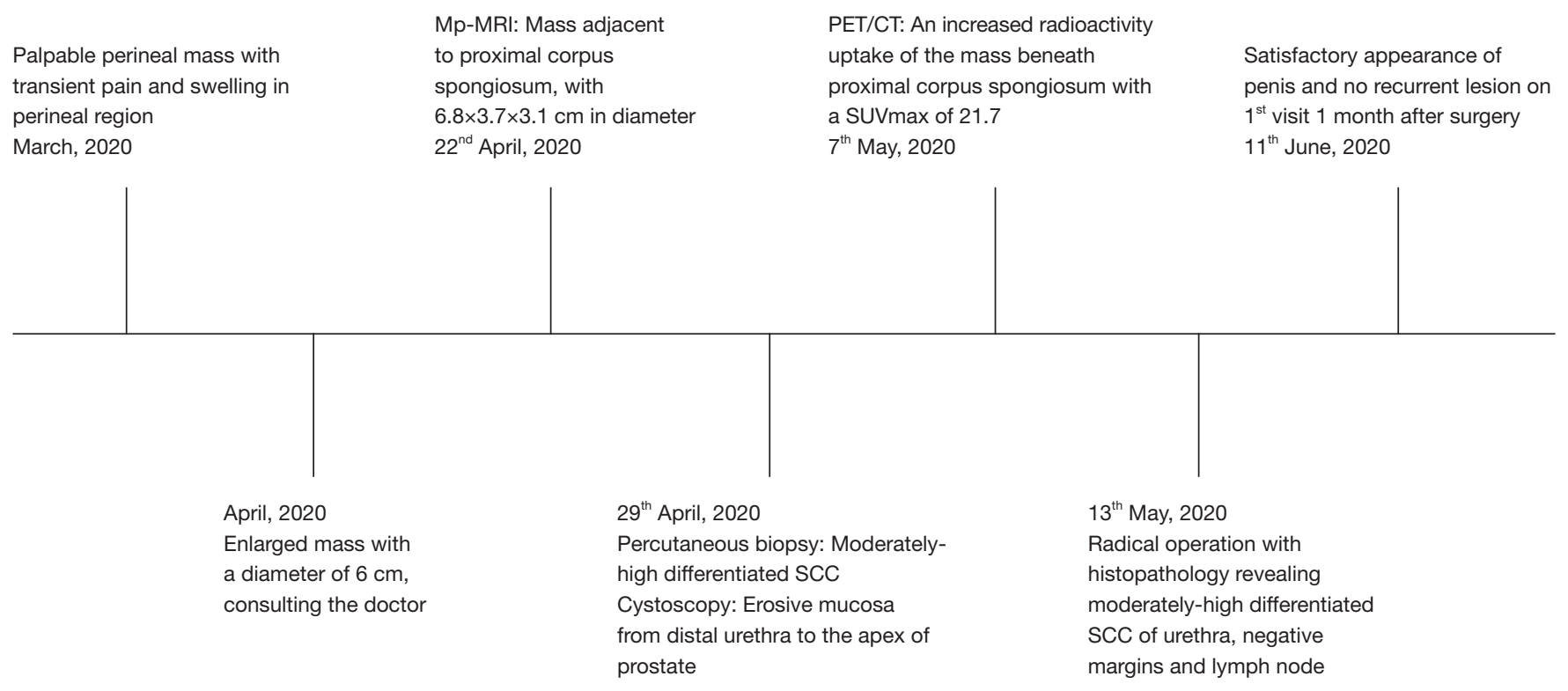

Figure 5 Timeline of the patient with SCC involving both distal and proximal urethra. mp-MRI, multiparametric magnetic resonance imaging; PET/CT, positron emission tomography/computed tomography; SCC, squamous cell carcinoma.

extra-urethral mass, bladder outlet obstruction, pelvic pain, urethrocutaneous fistula, abscess formation, and dyspareunia were reported progressive symptoms (12). For tumor located in the bulb or proximal urethra, dysuria was often reported due to the obstruction of urethra $(13,14)$. In this case, the patient presented with untypical symptoms of palpable mass and a transient pain in perineal region with frequent and painful urination. Moreover, the tumor had an unusual outward-infiltration inclination with no urethral stricture on cystoscopy, causing no dysuria during the course of the disease. The rare manifestation gave confusing information, leading to a percutaneous biopsy rather than biopsy under cystoscopy initially based on the suspicion of skin tumor, which indicated that patients with PUC may present with some unspecific symptoms even when advanced diseases have been developed. Those patients should not be missed at first visit.

In this case, we chose PET/CT rather than CT scan for the assessment of metastatic disease given patient's preference and the relatively better diagnostic efficiency. However, the enlarged LN on PET/CT is not in consistent with the histopathological result. Previous study showed a very high accuracy (up to 93\%) of MRI for clinical nodal staging to predict pathological LN involvement (11) and the use of pelvic MRI to assess the local extent of tumor and regional LN enlargement is highly recommended in the current guidelines (6). In contrast, a recent meta-analysis reported an only $57 \%$ sensitivity of PET/CT in detecting LN metastasis in clinically negative groin, and using PET/ CT in patients with distal urethral SCC with clinically negative groins was not recommended $(15,16)$. In the current study, a false positive result was shown on PET/CT, probably indicating a potentially unsatisfactory specificity of PET/CT. Despite all the facts above, we considered that the sensitivity and specificity of PET/CT and MRI for detecting LN metastasis in patients with PUC still needs further evaluation. Considering that the $\mathrm{N}$ stage has a close correlation with the prognosis $(9,16)$, which in some studies is even the only significant risk factors for overall survival (11), we thought a LND should be administered in patients with clinically suspected LN metastasis if a surgical intervention is considered, regardless of $\mathrm{T}$ stage. Prophylactic LND could be omitted as recommended in previous research (17).

PUC involving proximal urethra is believed to have a worse prognosis (18) but the evidence is sparse. Corbishley et al. suggested the distal and proximal urethral tumors should be separately designated with a site-specific staging and reporting system for the probable different tumor characteristics and treatment modality (3). In a populationbased study, Gakis et al. reported a rate of 54.1, 35.8 and $10.1 \%$ for tumor located in the proximal, distal, and proximal 
plus distal urethra, respectively (11). The EAU guidelines on PUC suggested a penile-preserving surgery as an alternative to penile amputation for male patients with distal PUC and additional iliac/inguinal LND for clinically suspected LN metastasis (level of evidence, LE: 3, strength rating: weak). And for treatment of UC of the prostate, an initial therapy of urethral-sparing approach with transurethral resection (TUR) and bacillus-Calmette Guerin (BCG) was recommended, with cystoprostatectomy and extended pelvic LND as a salvage approach or for patients with more advanced diseases (LE: 3, strength rating: strong) (6). However, treatment options for patients with PUC involving both distal and proximal urethra or other histology of prostatic urethra were not detailedly recommended in the current guidelines. Erin Bird and Michael Coburn (14) reported a series of three patients with invasive SCC located within the bulb and/ or penile urethra undergoing a surgical approach which they described as phallus preservation surgery. Penectomy/ urethrectomy was performed under a subcutaneous way with the glans preserved. Christopher et al. (19) reported a penile preservation combined with adjuvant chemo-radiotherapy on a patient with advanced bulbo-membranous SCC (pT4N0). The surgical approach included a nerve-sparing radical prostatectomy and urethrectomy with preservation of the glans and corpora cavernosa. Cadaveric tensor fascia lata was used to reconstruct the tunica of the ventral corpora cavernosa in order to preserve erectile function. In this case, the patient had a SCC involving both distal and proximal urethra with a large dimension (cT2N1M0). Inspired by the phallus preservation surgery, a transperineal-incision radical urethrectomy was performed to completely remove the tumor with the glans preserved. Due to the high risk of prostatic invasion and suspected pelvic $\mathrm{LN}$ metastasis, a laparoscopic prostatectomy combined with pelvic LND was performed simultaneously. Given the large dimension of tumor involving urethra, partial urethrectomy was precluded.

When it comes to the decision of treatment modality for PUC, the cosmetic, phycological, functional outcomes and patients' desire should also be taken into consideration along with oncologic principles (20). We considered the combination of tranperineal-incision urethrectomy and laparoscopic prostatectomy as an optimal approach with a good local clearance for patient with SCC involving both distal and proximal urethra, offering patient a chance of cure while respecting the wish to preserve a normal organ appearance. Moreover, once relapse occurred, further operation, radiation therapy and chemotherapy could be used as salvage or adjuvant therapy. The oncological outcomes for our approach in this case still needs longer follow-up time to confirm.

\section{Conclusions}

Patients with PUC involving both distal and proximal urethra may present with no symptoms of urethral stricture but only non-specific lower urinary symptoms. A transperineal-incision urethrectomy combined with prostatectomy we proposed in this case proves to be a safe and feasible approach to completely resect the tumor and preserve a normal appearance of penis, thus worth to be applied in the specific patient population.

\section{Acknowledgments}

The authors want to thank Chunmei Li from Dept. of Radiology, Hui Zhu from Dept. of Nuclear Medicine, Lei He and Wei Zhang from Dept. of Pathology, Beijing Hospital, for helping providing the imaging and pathologic data.

Funding: This research was supported by Beijing Hospital Clinical Research 121 Project (BJ-2018-090) and Innovation Funds for Postgraduates of Peking Union Medical College (No. 2019-1002-87).

\section{Footnote}

Reporting Checklist: The authors have completed the CARE reporting checklist. Available at http://dx.doi.org/10.21037/ tau-20-984

Conflicts of Interest: All authors have completed the ICMJE uniform disclosure form (available at http://dx.doi. org/10.21037/tau-20-984). The authors have no conflicts of interest to declare.

Ethical Statement: The authors are accountable for all aspects of the work in ensuring that questions related to the accuracy or integrity of any part of the work are appropriately investigated and resolved. All procedures performed in studies involving human participants were in accordance with the ethical standards of the Institutional Review Board of Beijing Hospital and with the Helsinki Declaration (as revised in 2013). Written informed consent was obtained from the patient.

Open Access Statement: This is an Open Access article distributed in accordance with the Creative Commons 
Attribution-NonCommercial-NoDerivs 4.0 International License (CC BY-NC-ND 4.0), which permits the noncommercial replication and distribution of the article with the strict proviso that no changes or edits are made and the original work is properly cited (including links to both the formal publication through the relevant DOI and the license). See: https://creativecommons.org/licenses/by-nc-nd/4.0/.

\section{References}

1. Gatta G, van der Zwan JM, Casali PG, et al. Rare cancers are not so rare: the rare cancer burden in Europe. Eur J Cancer 2011;47:2493-511.

2. Swartz MA, Porter MP, Lin DW, et al. Incidence of primary urethral carcinoma in the United States. Urology 2006;68:1164-8.

3. Corbishley CM, Rajab RM, Watkin NA. Clinicopathological features of carcinoma of the distal penile urethra. Semin Diagn Pathol 2015;32:238-44.

4. Abudurexiti M, Wang J, Shao N, et al. Prognosis of rare pathological primary urethral carcinoma. Cancer Manag Res 2018;10:6815-22.

5. Sui W, RoyChoudhury A, Wenske S, et al. Outcomes and Prognostic Factors of Primary Urethral Cancer. Urology 2017;100:180-6.

6. Gakis G, Witjes JA, Compérat E, et al. EAU guidelines on primary urethral carcinoma. Eur Urol 2013;64:823-30.

7. Riley DS, Barber MS, Kienle GS, et al. CARE guidelines for case reports: explanation and elaboration document. J Clin Epidemiol 2017;89:218-35.

8. Visser O, Adolfsson J, Rossi S, et al. Incidence and survival of rare urogenital cancers in Europe. Eur J Cancer 2012;48:456-64.

9. Aleksic I, Rais-Bahrami S, Daugherty M, et al. Primary

Cite this article as: Wang $M$, Yang $M$, Wu P, Deng S, Wang J, Chen J, Wang J, Liu M. Transperineal-incision urethrectomy combined with laparoscopic prostatectomy for a male patient with squamous cell carcinoma involving distal plus proximal urethra and untypical symptoms-a case report. Transl Androl Urol 2021;10(2):976-982. doi: 10.21037/tau-20-984 urethral carcinoma: A Surveillance, Epidemiology, and End Results data analysis identifying predictors of cancerspecific survival. Urol Ann 2018;10:170-4.

10. Rabbani F. Prognostic factors in male urethral cancer. Cancer 2011;117:2426-34.

11. Gakis G, Morgan TM, Efstathiou JA, et al. Prognostic factors and outcomes in primary urethral cancer: results from the international collaboration on primary urethral carcinoma. World J Urol 2016;34:97-103.

12. Gheiler EL, Tefilli MV, Tiguert R, et al. Management of primary urethral cancer. Urology 1998;52:487-93.

13. Krukowski J, Czajkowski M, Kłącz J, et al. Primary urethral carcinoma - unexpected cause of urethral stricture. Case report and review of the literature. Med Ultrason 2019;21:494-6.

14. Bird E, Coburn M. Phallus preservation for urethral cancer: subcutaneous penectomy. J Urol 1997;158:2146-8.

15. Ottenhof SR, Leone AR, Horenblas S, et al. Advancements in staging and imaging for penile cancer. Curr Opin Urol 2017;27:612-20.

16. Werntz RP, Riedinger CB, Fantus RJ, et al. The role of inguinal lymph node dissection in men with urethral squamous cell carcinoma. Urol Oncol 2018;36:526.e1-.e6.

17. Dayyani F, Hoffman K, Eifel P, et al. Management of advanced primary urethral carcinomas. BJU Int 2014;114:25-31.

18. Dalbagni G, Zhang ZF, Lacombe L, et al. Male urethral carcinoma: analysis of treatment outcome. Urology 1999;53:1126-32.

19. Christopher N, Arya M, Brown RS, et al. Penile preservation in squamous cell carcinoma of the bulbomembranous urethra. BJU Int 2002;89:464-5.

20. Karnes RJ, Breau RH, Lightner DJ. Surgery for urethral cancer. Urol Clin North Am 2010;37:445-57. 